\title{
Q-REG+: THE INNOVATIVE APPROACH TO ELECTRODE REGULATION*
}

\author{
Davide Onesti ${ }^{1}$ \\ Manuele Piazza² \\ Marco Ometto ${ }^{3}$
}

\begin{abstract}
Electrode regulation is the technology at the heart of the $A C$ and DC Electric ArC Furnace. As such, in the last decade it underwent continuous improvement to maximize the EAF performances, in terms of process efficiency, machine utilization and operator safety. Given this background, Danieli Automation's efforts in the field of electrode regulation have been focused on fast response to scrap cave-in, compensation of supply variations, automatic resonance detection, transformer protection, dynamic regulation of the electrical and chemical set-points, coordination and control of slagging and foaming agents, arc irradiance supervision. An extensive analysis of operator utilization and feedback was also carried out to develop an innovative system HMI. In this field, the choice of the variables shown to the user is the key aspect to allow a prompt process evaluation by the operator, who can refer to a condensed set of useful data. This paper will describe the innovative features and embedded models of the electrode regulation module (Q-REG+) and the supervision $\mathrm{HMI}$, together with the effects of their application on EAF process efficiency and system usability.
\end{abstract}

Keywords: Electrode Regulation, EAF, Dynamic Process Control, HMI.

Senior Engineer, Melt Shops, Business Unit Steel Making, Danieli Automation S.p.A, Buttrio, Italy. $R \& D$, Danieli Automation Research Center, Danieli Automation S.p.A, Buttrio, Italy.

Executive Vice President, Business Unit Steel Making \& Danieli Automation Research Center, Danieli Automation S.p.A, Buttrio, Italy. 


\section{ELECTRODE REGULATION IN THE STEEL MAKING PROCESS}

EAF steelmaking is an energy- and resource-intensive process that underwent significant modifications in the last decades. Numerous innovations, in the field of vessel design, slagging and stirring practices, transformer operation and DC technologies to name a few, have significantly reduced the energy and time requirements of the melting process. As a matter of fact, if in 1985 the best EAF performances were, on average, $500 \mathrm{kWh} / \mathrm{t}$ of electrical energy, electrode consumption equal to $4 \mathrm{~kg} / \mathrm{t}$ and a tap-to-tap time (TTT) of 120 minutes, currently the EAF state of the art is $330 \mathrm{kWh} / \mathrm{t}$, electrode consumption down to $1 \mathrm{~kg} / \mathrm{t}$ and a TTT of just 36 minutes [1].

The electric energy requirement of an EAF-based melt shop is largely connected to the melting process (Figure 1) and even limited improvements in this field translate into considerable yearly savings for the steel maker, in terms of electric energy and electrode consumption.

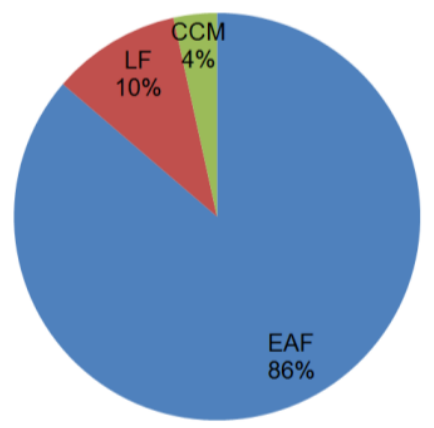

Figure 1. Fractions of the electric energy consumptions in a typical scrap-based EAF melt shop.

The electrode regulation system is the technology at the heart of the EAF and plays a very important role in the melting process, as it manages more than $50 \%$ of the process power input via the electric arcs. Typically, the system is a servo-hydraulic positioner, which controls the arc length acting on the position of each column. Primary $\mathrm{V}$ and $\mathrm{I}$ acquisition is performed on a small time scale (few microseconds) to allow a proper response to the fast-changing operating conditions inside the furnace.

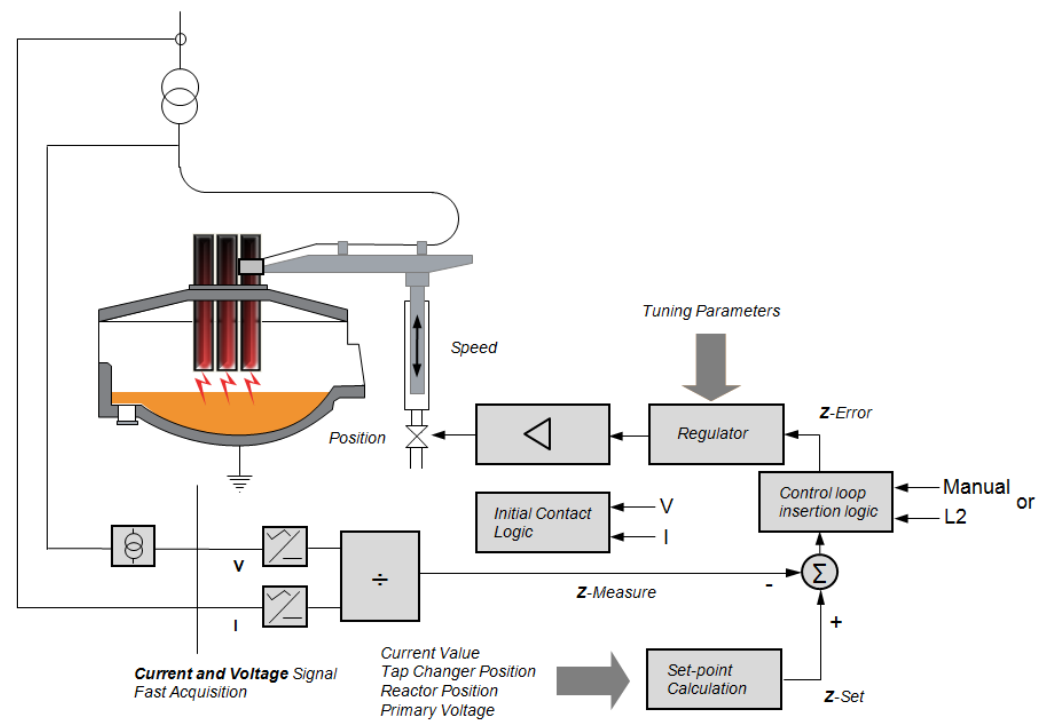


Figure 2. Impedance (Z) control block diagram.

The most used control strategies are based on arc impedance ( $Z$, Figure 2$)$, voltage (V) or current (I); the choice between them usually considers different factors:

- $\quad$ Desired maximum active power transferred to the melt.

- $\quad$ Melting process progress.

- $\quad$ Needed control stability.

- $\quad$ Tolerance on transformer technical limits.

- $\quad$ EAF practice.

The most common controller architecture for AC electrode regulation is MISO (Multiple Input - Single Output), meaning that, typically, the three phases are controlled separately. From an electrical point of view though, the phases are electrically interdependent, as the working electrical parameters of each one influence the other two. A suitable electrical model which takes into account the mutual effects between the electric phases can be included in an advanced MIMO (Multiple Input - Multiple Output) electrode regulator control. The most significant benefits of such an approach are the full monitoring and control of the furnace electric balancing; this allows a lower control response time and robustness opening up to several advanced control features like controlled unbalanced operation (useful for example to lower the thermal load on a specific EAF panel).

Electrode lowering movement is controlled by the counter pressure exerted by the hydraulic system. An advanced electrode regulation system performs the regulation of this parameter too; the aim is to keep a constant oil return pressure and avoid electrode breakage risk in case of considerable amount of non conductive material under the electrode tip.

The initial boring-down phase is usually associated with high arc instability and potential EAF roof damage; its duration depends mainly on the charge average dimensions and distribution. The ability to detect when this initial phase has come to an end, monitoring electric parameters and electrode tip position, allows to automatically increase the furnace power as soon as possible and increase furnace utilization factor.

Voltage variations of the electric supply can occur during EAF operation due to its high load on the network. Automatic compensation of these variations ensures uniform operation without operator intervention. Together with the ability to perform over-current protection, thermal protection and secondary isolation control, these regulator features underline the importance of a proper electrode control on the plant and also on the network itself.

The real-time fast $\mathrm{V}$ and $\mathrm{I}$ data acquisition and processing also allows the arc status evaluation, which is crucial to ensure a proper foamy slag practice. A proprietary and robust transfer function, based on Fast Fourier Transform of the electric signals, is typically used to qualify the arcs coverage ( $\mathrm{ACl}$, Arc Coverage Index) and thus control both chemical and electrical process set-points (Figure 3). 

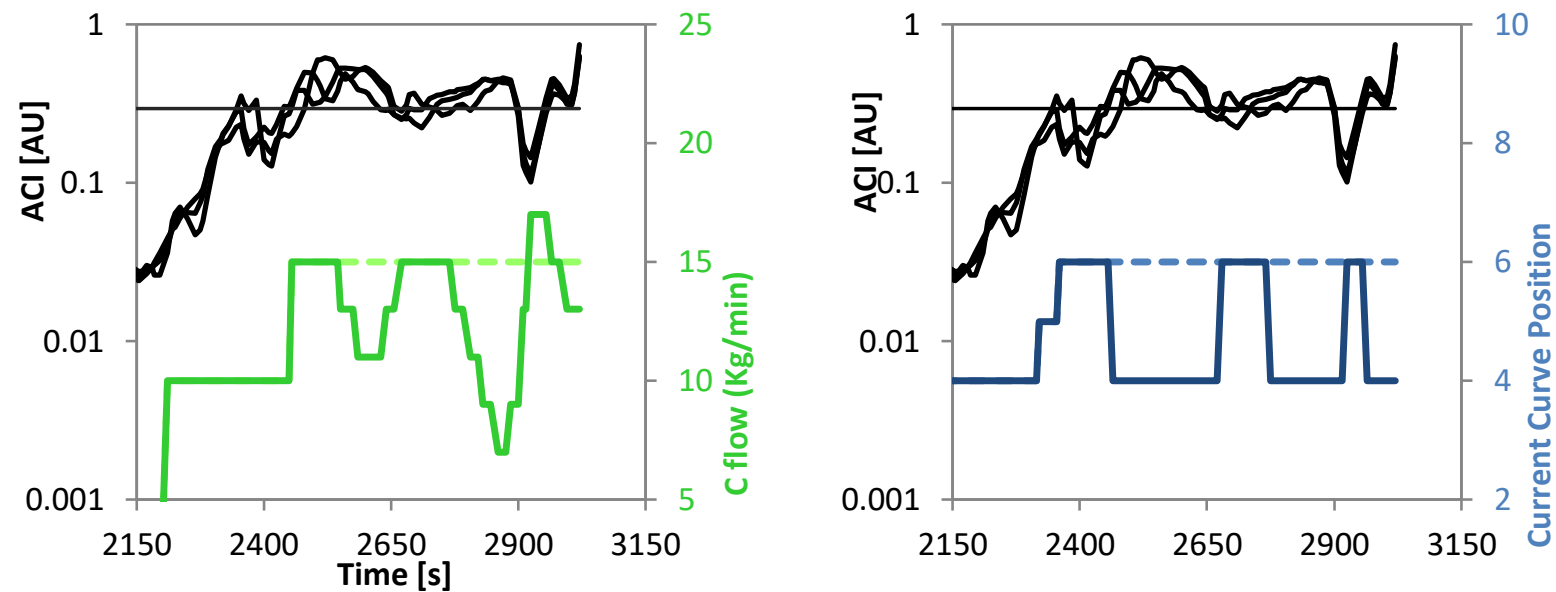

Figure 3. (a) $C$ injection regulation and (b) current curve regulation based on Arc Coverage Index $(\mathrm{ACl})$.

In favorable conditions, arc coverage is detected to be over a specific threshold; the dynamic modification of current and voltage is thus enabled and performed to maximize long arc operation, to reduce electric power losses and electrode tip consumption. Dynamic regulation of the carbon flow optimizes its consumption, by reducing the corresponding static set-point it when the arc is covered.

Proper $\mathrm{C}$ injection and slag foaming management must be performed on DC furnaces as well. In this case, signal harmonic analysis is not available and the control strategy is based on the acquisition of structure vibration or machine noise. The signal is in very good accordance with its harmonic analysis counterpart and a similar control strategy can thus be implemented on DC EAFs.

\section{Q-REG+ TECHNOLOGICAL PACKAGE}

2.1 Hardware configuration
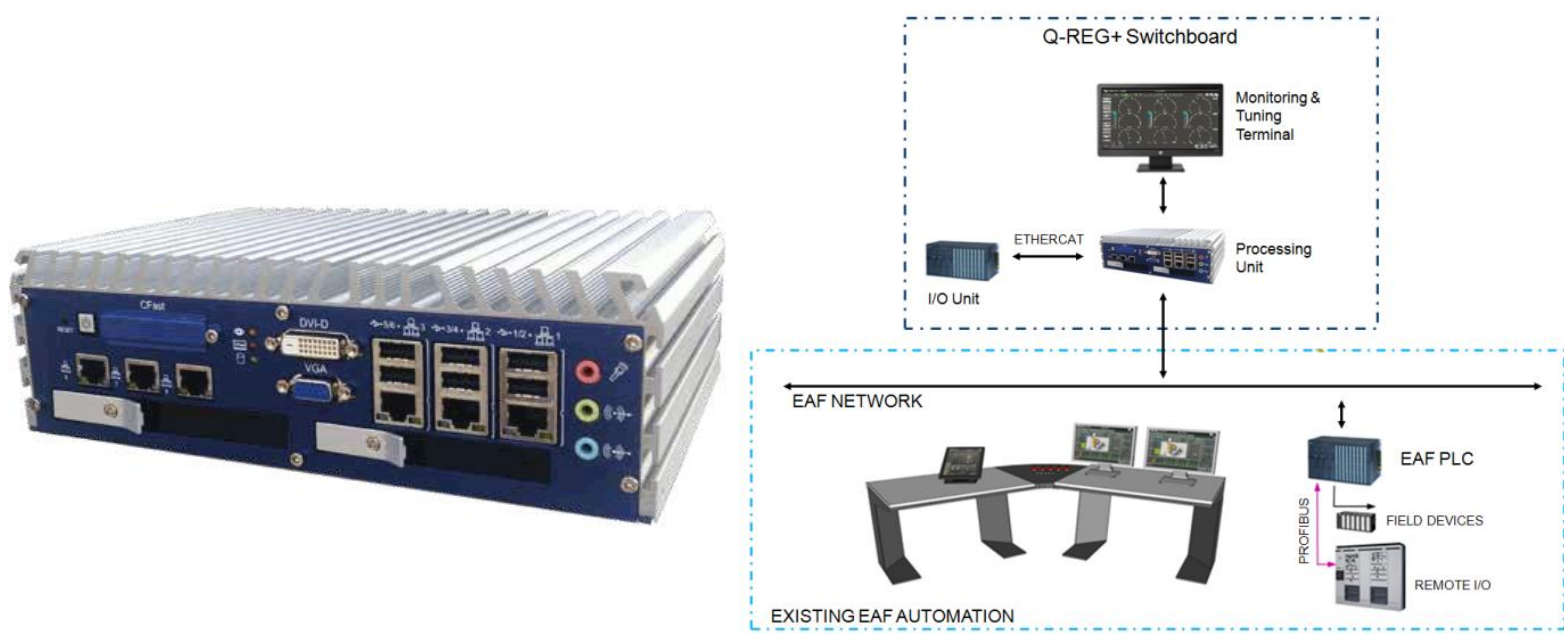

Figure 4. (a) Q-REG+ processing unit and (b) HW configuration.

To streamline and simplify the electrode regulator architecture, a complete system porting to a single platform was performed. From a hardware point of view, the DA-PAC (Programmable Automation Controller) platform is based on highperformance commercial processing unit which ensure plenty of performance (quad- 
core i7-based) and the ruggedness needed for plant operation (fanless, $-25 \div 70{ }^{\circ} \mathrm{C}$, $10 \% \div 95 \%$ humidity operative range). The overall dimensions are also relatively limited as no additional PLC components are required. Thanks to the system added simplicity, interaction with several different PLC architectures is immediately available.

\subsection{Supervision HMI}
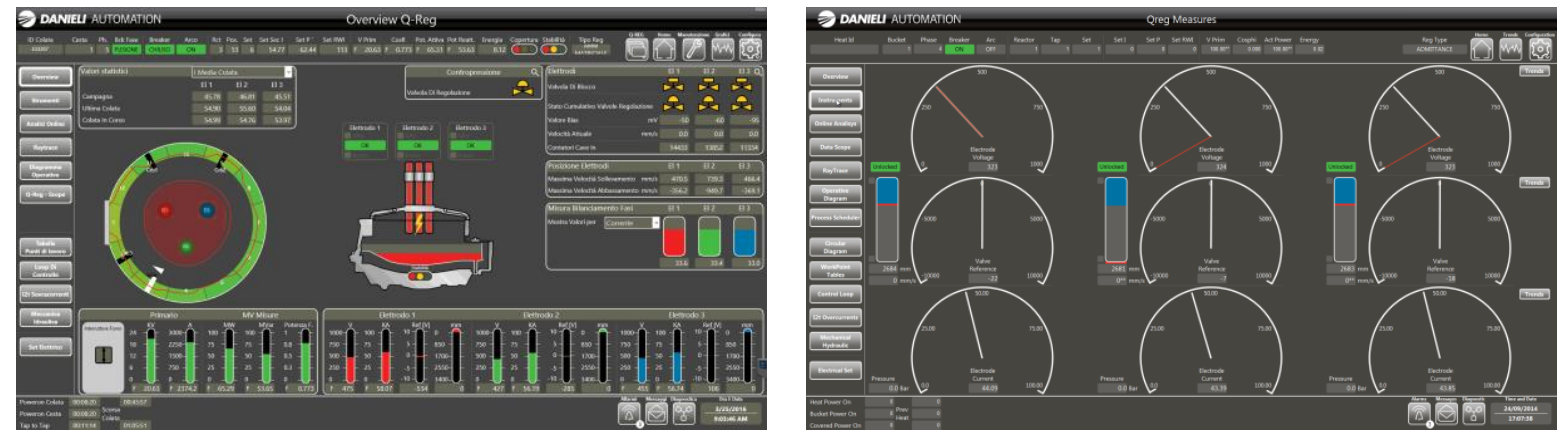

Figure 5. Electrode regulator overview and electric measures dashboard.

The supervision HMI was developed in COPA-DATA Zenon environment. After substantial testing and feedback from the users, a limited amount of often-used pages were selected to be called from the main page, to avoid interface cluttering and enhance HMI usability (Figure 5). The interface was developed with touchscreen operation in mind because of its full integration in Danieli Q3 Technologies and machine pulpit design.
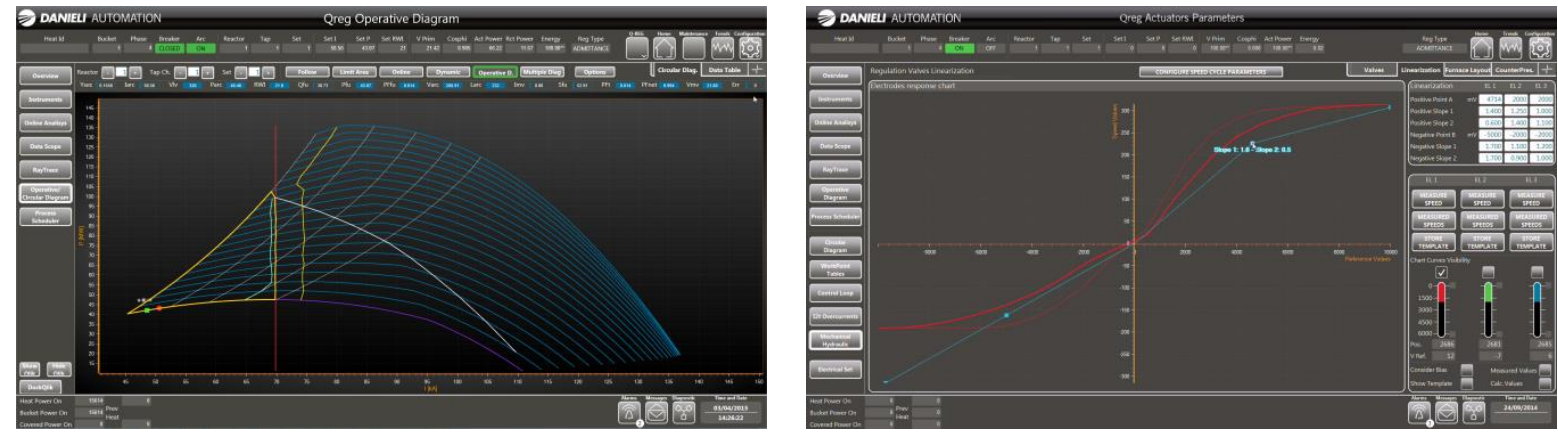

Figure 6. (a) Interactive circular diagram set-point input and (b) valve linearization.

The ability to visualize the furnace work area and electric set-points on the operative circular diagram allows a quick EAF operation evaluation and fast machine finetuning (Figure 6), according to numerous different process parameters (Arc V, I, length and power factor, among others).

The hydraulic system response nonlinearity is measured automatically and visualizes measured and calculated values after the test.

Electrode linearization function can be used, for instance, to compensate the effect of counter pressure regulation that makes the descent speed slightly lower than the lifting one. The automatic valve linearization speeds up the tuning process and allows machine response modification by the user. Automatic bias detection and electrode speed difference detection were also included to enhance the drift detection and the diagnostic of the servovalves. Automatic detection of mechanical resonance frequency is being implemented to tune the notch filter according to the column-arm- 
electrode system rigidity: the aim is to avoid resonance phenomena and to dynamically adapt to the changes of the system response during furnace operation.

A complete suite of diagnostic tools (Q-REG SCOPE, Figure 7) is included in the technological package and run on the same processing unit of the regulator. Advanced machine monitoring and statistical analysis allow to trend multiple process variables (power factor, arc voltage, arc current, arc power, refractory consumption index) filtering them on several practice parameters. $X-Y$ dispersion graphs and histogram distribution plots quickly identify electrodes mechanical response deviations and the distribution of electrical variables or control parameters.

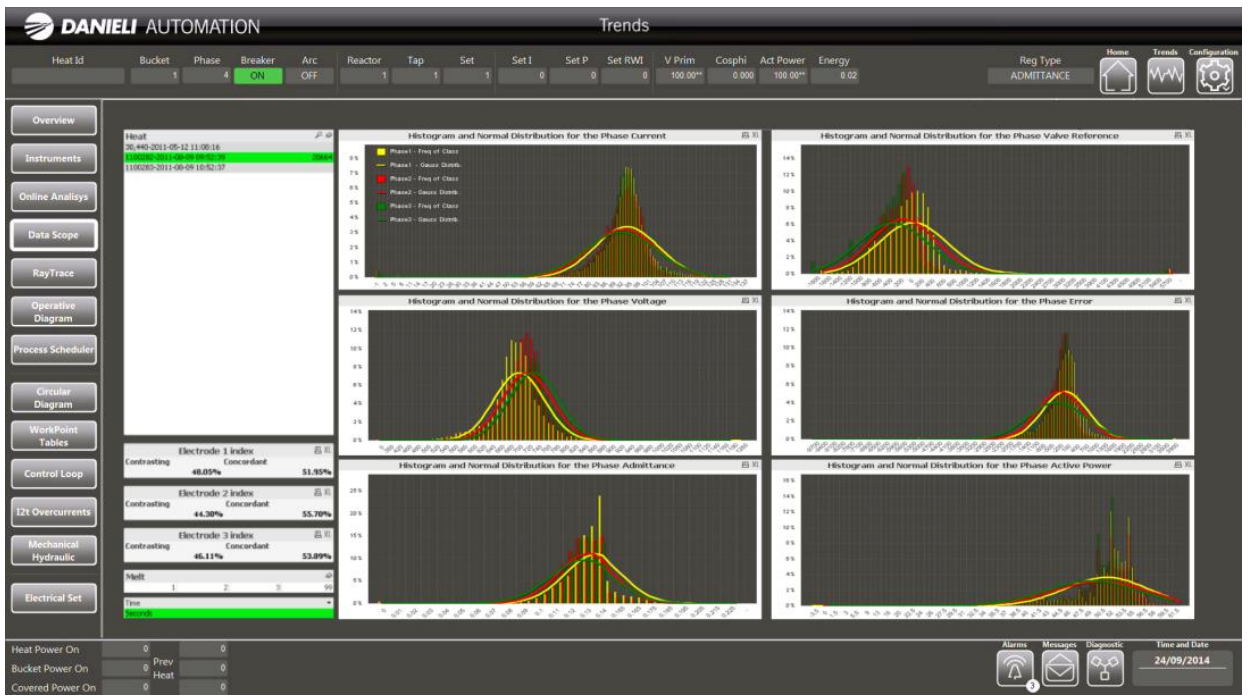

Figure 7. Process variables distribution analysis.

\subsection{Electrode Irradiance Supervisor (Q-RAY TRACE)}

An innovative electrode irradiance model was also included to track the radiation power on the furnace walls (Figure 8). The radiative power of each arc is calculated and the thermal radiation power along the EAF walls is plotted in real time to the operator (red polar trend). With proper output filtering and integration, the irradiance is thus displayed versus the recorded panels temperature (green polar histograms) to verify immediately the consequences of eventual unbalanced operation on the cooling system.

Such a virtual variable allows to implement the electric set-point modification considering also the thermal load on the panels. Nonetheless, even without a coordinating L2 automation system, the regulator is able to adapt to different process steps thanks to its integrated process scheduler, so a modular approach still remains usable and advantageous when the available resources are limited. 


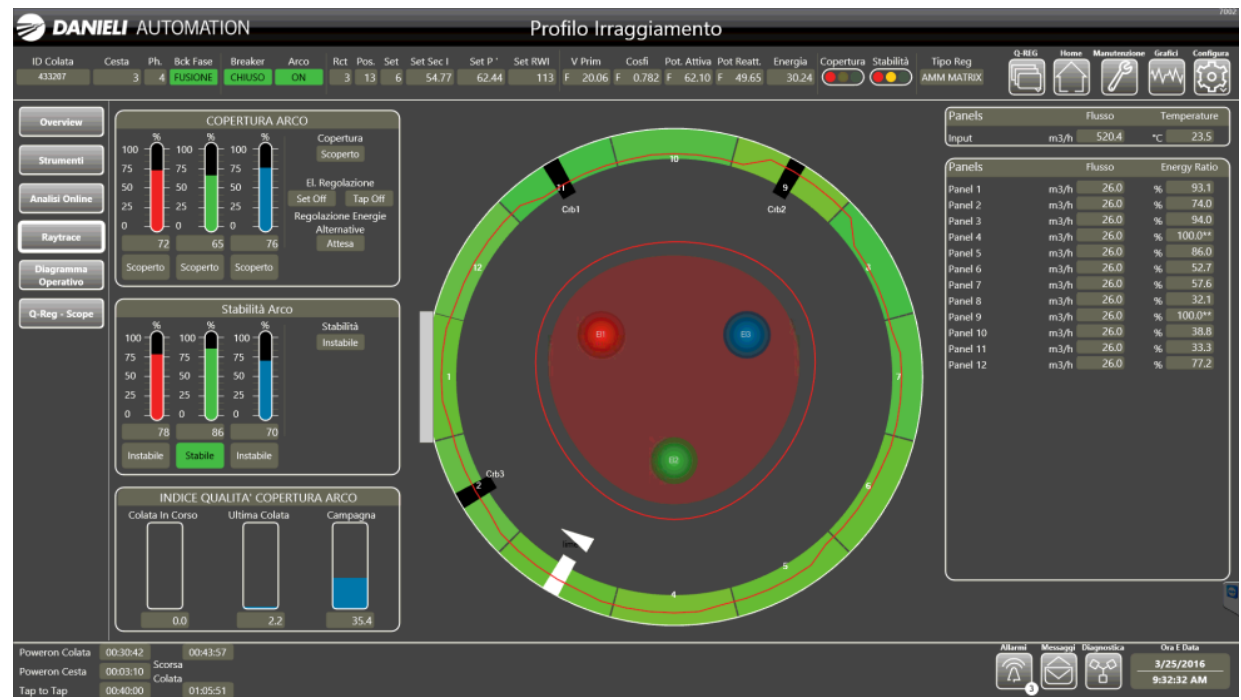

Figure 8. Electrode irradiance and cooling panels temperature dedicated view.

\section{OPERATIVE RESULTS}

To validate the innovative MIMO (matrix-based) control strategy, an extensive test campaign was carried out to compare the performances of the new control to its previous releases. Specifically, the 3 controls compared are:

- PLC-based MISO (Hireg)

- PAC-based MISO (Q-Reg)

- PAC-based MIMO (Q-Reg Matrix)

Specific energy (Lower is better)

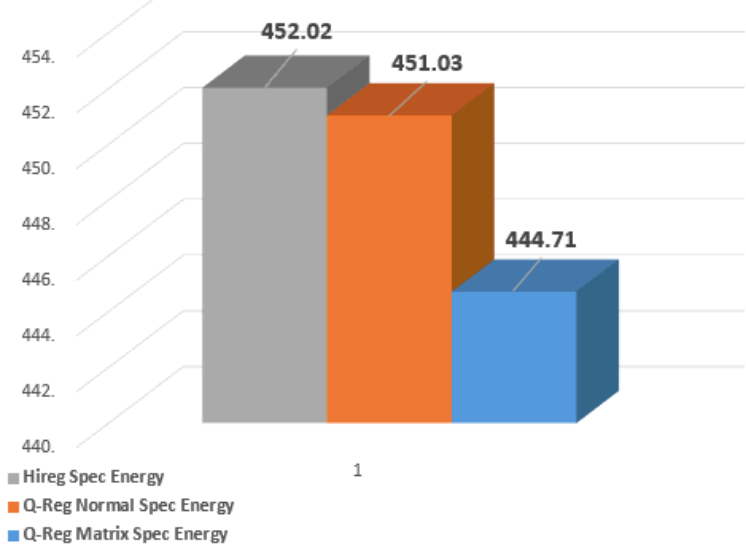

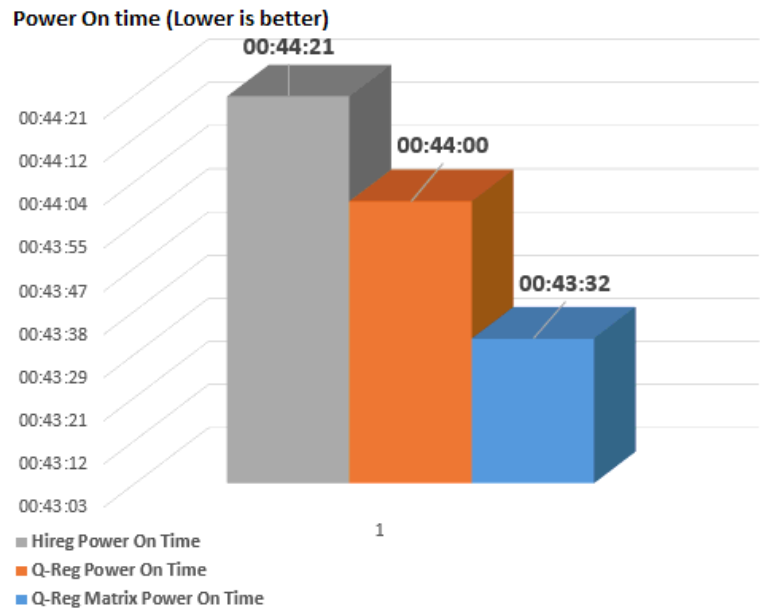

Figure 9. Operational results comparison between PLC MISO, PAC MISO and PAC MIMO electrode regulation.

The operational results (Figure 9) show that the high-performance PAC control platform increases the process performances in terms of power-on time and, to a lower extent, the specific energy consumption. Q-Reg Matrix, on the other hand, improves significantly both the specific energy consumption $(-7.3 \mathrm{kWh} / \mathrm{t})$ and the power-on time (-0.8 min).

\section{CONCLUSION}


The intense porting and re-engineering activity on the traditional electrode regulator architecture showed several advantages in terms of system setup time and machine operability. EAF operators showed positive feedback regarding the innovative interface and the radiation monitoring features, proving that a synthesis of the most important process variables is an efficient approach to machine status display.

The regulator control architecture continues to improve furnace performances: its latest release, which includes an MIMO control strategy and an electrical phases coupling model, improved significantly both the specific energy consumption and the power-on time, proving that a high-performances platform and advanced control strategies lead to important savings for the steelmaker.

\section{REFERENCES}

1 J. I. A. Albizun, H. Schult, "Benchmark analysis - A Tool to Define the Next Development Steps?", SGL Group 